\title{
Integrated Deterministic and Probabilistic Safety Analysis for Safety Assessment of Nuclear Power Plants
}

\author{
Francesco Di Maio, ${ }^{1}$ Enrico Zio, ${ }^{1,2}$ Curtis Smith, ${ }^{3}$ and Valentin Rychkov ${ }^{4}$ \\ ${ }^{1}$ Energy Department, Politecnico di Milano, Via La Masa 34, 20126 Milano, Italy \\ ${ }^{2}$ Chair on System Science and Energetic Challenge, Fondation EDF, Centrale Supélec, Chatenay-Malabry Cedex, 92295 Paris, France \\ ${ }^{3}$ Idaho National Laboratory, Idaho Falls, ID 83415, USA \\ ${ }^{4}$ EDF ReD, 92141 Clamart Cedex, France
}

Correspondence should be addressed to Francesco Di Maio; francesco.dimaio@polimi.it

Received 28 June 2015; Accepted 6 July 2015

Copyright (C) 2015 Francesco Di Maio et al. This is an open access article distributed under the Creative Commons Attribution License, which permits unrestricted use, distribution, and reproduction in any medium, provided the original work is properly cited.

The present special issue provides an overview of the research in the field of Integrated Deterministic and Probabilistic Safety Assessment (IDPSA) of Nuclear Power Plants (NPPs).

Traditionally, safety regulation for NPPs design and operation has been based on Deterministic Safety Assessment (DSA) methods to verify criteria that assure plant safety in a number of postulated Design Basis Accident (DBA) scenarios. Referring to such criteria, it is also possible to identify those plant Structures, Systems, and Components (SSCs) and activities that are most important for safety within those postulated scenarios. Then, the design, operation, and maintenance of these "safety-related" SSCs and activities are controlled through regulatory requirements and supported by Probabilistic Safety Assessment (PSA).

On the other hand, compliance with the evolving regulatory requirements and the drive for enhancements in safety and economics calls for the development of innovative deterministic and probabilistic approaches of assessment for the existing NPPs and their life extension. In this respect, a medium-term challenge is to combine the use of deterministic and probabilistic methodologies in an IDPSA framework, which includes the tools and methods that couple probabilistic and deterministic approaches.

In this special issue, this coupling is shown to

(i) improve the computational approach of NPPs Thermal-Hydraulic (TH) models, thanks to the employment of metamodels for multiparameter and nonlinear modelling and for the throughout exploration of the space of combinatorial plant scenarios. Gaussian
Process- (GP-) based surrogate models are proposed for incorporating new information from tests or operating experience into $\mathrm{TH}$ codes, for reducing the uncertainty in the output of the code and for dealing with the computational burden required for calibrating parameters of the $\mathrm{TH}$ codes used for the deterministic simulation of the NPPs response in accident scenarios,

(ii) treat aleatory (stochastic aspects of accident scenarios) and epistemic (model and parameters) uncertainties in a consistent manner. A Risk Informed Safety Margin Characterization (RISMC) approach is proposed for treating the uncertainties (e.g., stochastic for the safety system recovery times and epistemic for the model parameters) for a joint quantification of the frequency of core damage probability and of the safety margin for a relevant NPP accident,

(iii) identify and characterize a priori unknown vulnerable scenarios. Two approaches are proposed: a characterization of a failure domain based on scenario clustering by decision trees, with the goal of highlighting the influence of timing and order of events in the scenario grouping thereby obtained and a risk-based clustering method for the identification of Prime Implicants (PI) and of Near Misses (NM) scenarios, that is, minimum vulnerable combinations of failure events that lead the system to fault or quasi-fault states (a condition close to accident), respectively, 
(iv) describe the time-dependent and spatial interactions between physical phenomena, equipment failures, safety and nonsafety systems interactions, control logic, and operator actions. An improved model to describe the time-dependent interactions between fire and firefighting by simultaneously accounting for stochastic and epistemic uncertainties by a Fire Dynamics Simulator (FDS) embedded into a Monte Carlo Dynamic Event Tree (MCDET) is proposed. Also, a combined modelling approach based on the Theory of Probabilistic Dynamics (TPD) for the dynamic reliability modelling and simulation of an accident under uncertain events is proposed for the assessment of the probability of a NPP containment failure due to hydrogen combustion,

(v) reduce the reliance on expert judgment and simplifying (or overly conservative) assumptions about interdependencies. A deterministic analysis is integrated with a probabilistic analysis for the estimation of a NPP core damage frequency, with as little as possible inclusion of expert judgment into the assumption adoption. Also, highlighting the effects of decoupling, at different level of interdependence, the analysis of a NPP liquefaction from a ground vibration motion analysis due to different expert judgment on their mutual interaction is given,

(vi) reduce the overall costs of the analysis and reduce the maintenance costs of the integrated models compared to separate deterministic and probabilistic models. The benefit of an explicit treatment of timedependencies and priorities of defects/failure occurrences by integrated deterministic and probabilistic models is shown for an improved planning of the maintenance activities on NPP components.

As this journal aims to promote development in the area of nuclear sciences and technologies, it has been natural to present this special issue reporting on recent research in the field of IDPSA in support to the sustainability and safety of the nuclear option and the renaissance of nuclear technology in the world and especially in those countries where nuclear programs have not yet been developed.

\section{Acknowledgments}

Finally, we wish to point out that this special issue would have not been possible without the outstanding contributions of all the authors and reviewers. Our sincere professional appreciation and personal gratitude go to all these people.

Francesco Di Maio

Enrico Zio

Curtis Smith

Valentin Rychkov 


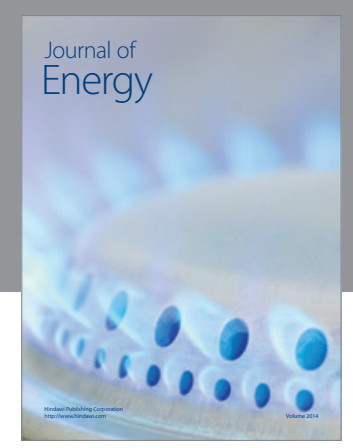

Journal of

Industrial Engineering
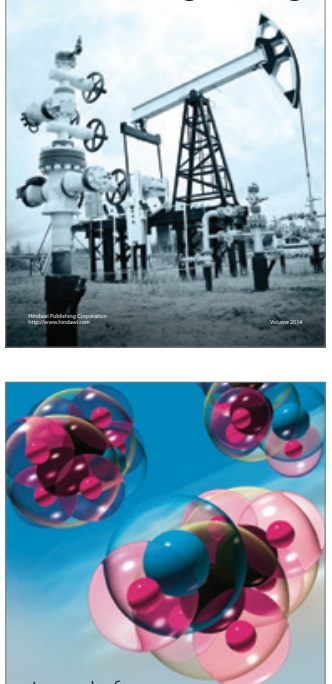

Fuels
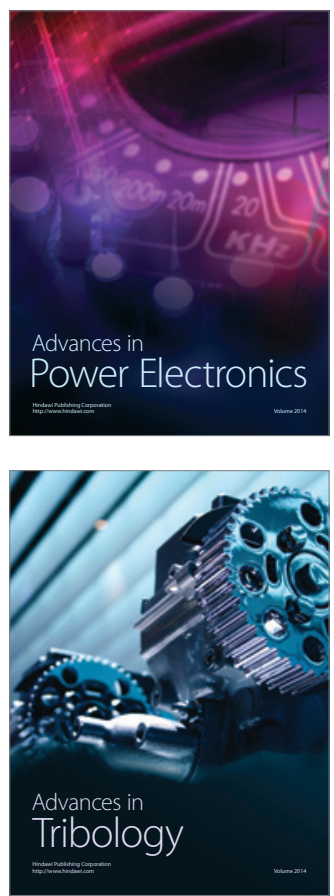

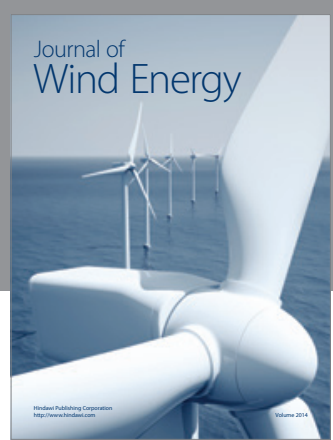

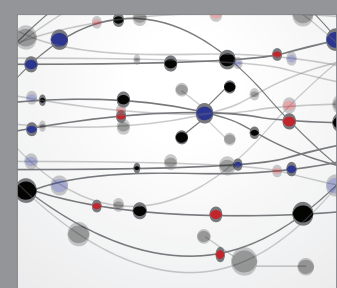

The Scientific World Journal

Submit your manuscripts at http://www.hindawi.com

Journal of

Structures
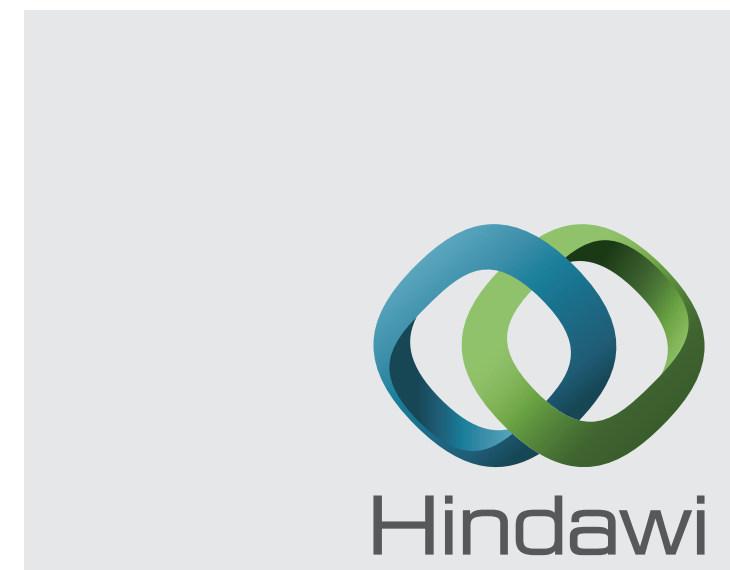

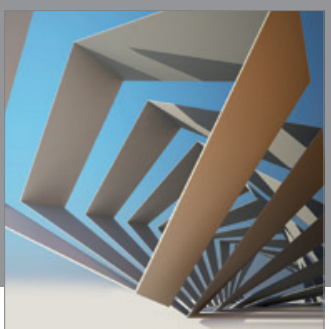

Rotating

Machinery
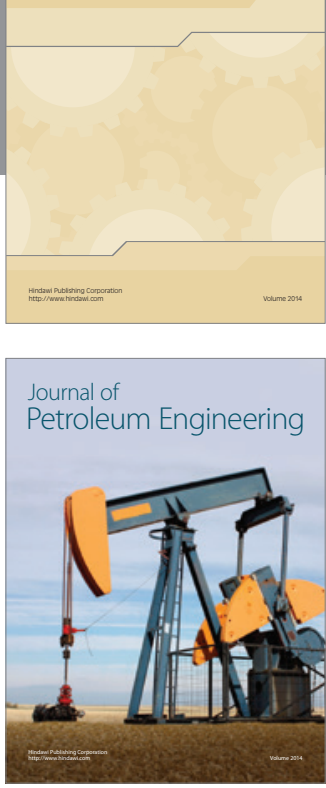

Journal of

Solar Energy
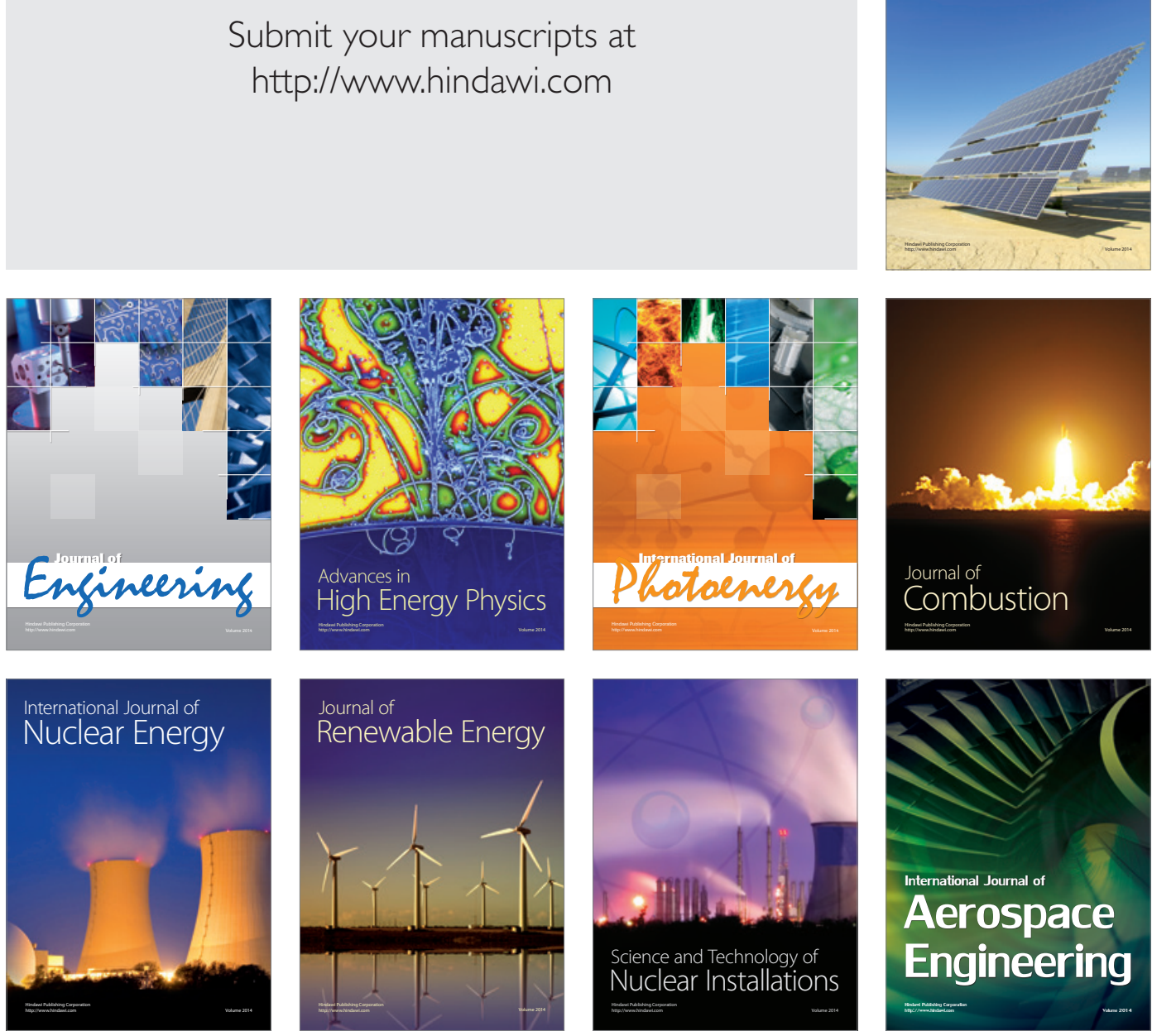